\title{
ON THE STEINBERG CHARACTER OF A FINITE SIMPLE GROUP OF LIE TYPE
}

\author{
BHAMA SRINIVASAN \\ (Received 4 March 1969; revised 16 July 1969) \\ To Bernhard Hermann Neumann on his 60th birthday \\ Communicated by G. E. Wall
}

\section{Introduction}

Let $K$ be an algebraically closed field of characteristic $p>0$. If $G$ is a connected, simple connected, semisimple linear algebraic group defined over $K$ and $\sigma$ an endomorphism of $G$ onto $G$ such that the subgroup $G_{\sigma}$ of fixed points of $\sigma$ is finite, Steinberg ([6], [7]) has shown that there is a complex irreducible character $\chi$ of $G_{\sigma}$ with the following properties. $\chi$ vanishes at all elements of $G_{\sigma}$ which are not semisimple, and, if $x \in G$ is semisimple, $\chi(x)= \pm n(x)$ where $n(x)$ is the order of a Sylow $p$-subgroup of $\left(Z_{G}(x)\right)_{\sigma}\left(Z_{G}(x)\right.$ is the centraliser of $x$ in $\left.G\right)$. If $G$ is simple he has, in [6], identified the possible groups $G_{\sigma}$; they are the Chevalley groups and their twisted analogues over finite fields, that is, the 'simply connected' versions of finite simple groups of Lie type. In this paper we show, under certain restrictions on the type of the simple algebraic group $G$ and on the characteristic of $K$, that $\chi$ can be expressed as a linear combination with integral coefficients of characters induced from linear characters of certain naturally defined subgroups of $G_{\sigma}$. This expression for $\chi$ gives an explanation for the occurence of $n(x)$ in the formula for $\chi(x)$, and also gives an interpretation for the \pm 1 occuring in the formula in terms of invariants of the reductive algebraic group $Z_{G}(x)$.

I am very grateful to Professor T. A. Springer for making available to me a preprint of [6].

\section{Notation and preliminary lemmas}

In this section, unless otherwise stated, $G$ denotes a connected reductive linear algebraic group defined over an algebraically closed field $K$ of characteristic $p>0$, and $\sigma$ an endomorphism of $G$ onto $G$ such that $G_{\sigma}$ is finite. $T$ is a maximal torus of $G$ such that both $T$ and a Borel subgroup contaning $T$ are both fixed by $\sigma ; T$ is unique up to conjugacy by an element of $G_{\sigma}$ (see [6]). $N$ is the normaliser of $T$ in $G$ and $N / T=W$. Then $\sigma$ induces an automorphism of the finite group $W$. 
The connected component of the identity of any closed subgroup $H$ of $G$ will be denoted by $H_{0}$. We refer to [1] for the relevant facts about algebraic groups. For any finite group $H,|H|$ denotes its order.

Definition. Two elements $n_{1}, n_{2} \in N$ are said to be $\sigma$-conjugate if there exists an element $n \in N$ such that $n_{2}=n n_{1} n^{-\sigma}$. For any $n \in N$ let

$Z(n)$ be the centraliser of $n$ in $N$,

$$
Z^{\prime}(n)=\left[n^{\prime} \in N \mid n^{\prime} n n^{\prime-\sigma}=n\right]
$$

and

$$
Z^{\prime \prime}(n)=\left[n_{0} \in N \mid n_{0}^{-1} n n_{0}=n^{\sigma}\right] .
$$

$Z^{\prime}(n)$ is a subgroup of $N$. $Z^{\prime \prime}(n)$ may be empty, but if there is an element $n_{0} \in Z^{\prime \prime}(n)$ then $Z^{\prime \prime}(n)=Z(n) n_{0}$. Let

$$
K(n)=\left[n^{\prime} \in Z(n) \mid n^{\prime} \text { centralises }(Z(n) \cap T)_{0}\right] .
$$

If $w \in W$, the subgroup $Z^{\prime}(w)$ and the subset $Z^{\prime \prime}(w)$ of $W$ are defined similarly as

and

$$
\begin{aligned}
Z^{\prime}(w) & =\left[w^{\prime} \in W \mid w^{\prime} \dot{w} w^{\prime-\sigma}=w\right] \\
Z^{\prime \prime}(w) & =\left[w_{0} \in W \mid w_{0}^{-1} w w_{0}=w^{\sigma}\right] .
\end{aligned}
$$

$Z(w)$ is the centraliser of $w$ in $W . K(w)$ is the subgroup of $W$ of all elements of $Z(w)$ leaving elementwise fixed the connected component of the identity of the subgroup of $T$ of fixed points of $w$.

We first state a result of Steinberg [6] which is a generalization of a wellknown result of Lang [3].

LEMMA 1. (Steinberg) Let $H$ be a connected linear algebraic group over $K$ and $\sigma$ an endomorphism of $H$ onto $H$ such that $H_{\sigma}$ is finite. Then the map $x \rightarrow x x^{-\sigma}$ of $H$ into $H$ is surjective.

Our aim now is to define certain 'Cartan subgroups' of $G_{\sigma}$ and to set up a one-to-one correspondence between the $G_{\sigma}$-conjugacy classes of these subgroups and $\sigma$-conjugacy classes of $W$. This correspondence is known in the case when $G$ is defined and split over a finite field of $q$ elements and $\sigma$ is the $q^{\text {th }}$ power map, but we have not been able to find a description of it in the literature.

Lemma 2. If two elements $n_{0}, n_{1} \in N$ are $\sigma$-conjugate modulo $T$, they are $\sigma$-conjugate in $N$.

Proof. Let $n_{1}=n n_{0} n^{-\sigma} t$ where $n \in N, t \in T$. By Lemma $1 n_{1}=a a^{-\sigma}$ where $a \in G$. Hence

$$
a^{-1} n n_{0} n^{-\sigma} a^{\sigma}=a^{-\sigma} t^{-1} a^{\sigma} .
$$

Since $n_{1}$ normalizes $T, a^{-\sigma} t^{-1} a^{\sigma} \in a^{-1} T a$. Also $a^{-1} T a$ is connected. Thus by Lemma 1 we can write

$$
a^{-\sigma} t^{-1} a^{\sigma}=a^{-1} t_{1} a a^{-\sigma} t_{1}^{-\sigma} a^{\sigma}=a^{-1} t_{1} n_{1} t_{1}^{-\sigma} a^{\sigma}
$$


for some $t_{1} \in T$. Together with (2.1) this yields $n_{1}=t_{1}^{-1} n n_{0} n^{-\sigma} t_{1}^{\sigma}$, showing that $n_{1}, n_{0}$ are $\sigma$-conjugate in $N$. This proves the lemma.

Suppose $n_{0} \in N$ and $n_{0}=a a^{-\sigma}(a \in G)$. Then the maximal torus $a^{-1} T a$ and its normaliser $a^{-1} N a$ are fixed by $\sigma$. Conversely if the maximal torus $a^{-1} T a$ is fixed by $\sigma$ then $a a^{-\sigma} \in N$. We investigate the $G_{\sigma}$-conjugacy of such maximal tori in the next lemma.

Lemma 3. Let $n_{0}, n_{1} \in N, n_{0}=a a^{-\sigma}, n_{1}=b b^{-\sigma}(a, b \in G)$. Then $a^{-1} T a$ and $b^{-1} T b$ are conjugate by an element of $G_{\sigma}$ if and only if $n_{0}, n_{1}$ are $\sigma$-conjugate in $N$ (and hence, by Lemma 2, if and only if $n_{0} T$ and $n_{1} T$ are $\sigma$-conjugate in $W$ ).

Proof. If $n_{0}=n n_{1} n^{-\sigma}(n \in N)$ then $a^{-1} n b=g \in G_{\sigma}$ and $g^{-1} a^{-1} T a g=$ $b^{-1} T b$. Conversely let $g^{-1} a^{-1} T a g=b^{-1} T b$ where $g \in G_{\sigma}$. Then $a g b^{-1}=n \in N$, and $n_{0}=n n_{1} n^{-\sigma}$.

It follows from Lemma 2 and 3 that to each maximal torus $a^{-1}$ Ta fixed by $\sigma$ there corresponds the element $\left(a a^{-\sigma}\right) T$ of $W$ which is unique up to $\sigma$-conjugacy in $W$. Furthermore there is a one-to-one correspondence between $G_{\sigma}$-conjugacy classes of maximal tori of $G$ fixed by $\sigma$ and $\sigma$-conjugacy classes of $W$. For these maximal tori $a^{-1} \mathrm{Ta}$ we now consider the subgroups $\left(a^{-1} \mathrm{Ta}\right)_{\sigma}$ ('Cartan subgroups' of $\left.G_{\sigma}\right)$ and the subgroups $\left(a^{-1} N a\right)_{\sigma}$.

Lemma 4. Let $a^{-1}$ Ta be a maximal torus fixed by $\sigma$, and let $a a^{-\sigma}=n_{0}$, $n_{0} T=w_{0} \in W$. Then

is isomorphic to $Z^{\prime}\left(w_{0}\right)$.

$$
\left(a^{-1} N a\right)_{\sigma} /\left(a^{-1} T a\right)_{\sigma}
$$

Proof. Let $a^{-1} n a \in\left(a^{-1} N a\right)_{\sigma}(n \in N)$ and consider the map $a^{-1} n a \rightarrow n T=w$ of $\left(a^{-1} N a\right)_{\sigma}$ into $W$. Then $w w_{0} w^{-\sigma}=w_{0}$ and hence $\left(a^{-1} N a\right)_{\sigma}$ is mapped into $Z^{\prime}\left(w_{0}\right)$. By the proof of Lemma $2\left(a^{-1} N a\right)_{\sigma}$ is actually mapped onto $Z^{\prime}\left(w_{0}\right)$ and this proves the lemma.

The next lemma is basic for our construction of the Steinberg character of $G_{\sigma}$.

LeMmA 5. Let $a_{i}^{-1} N a_{i}(i=1,2, \cdots)$ be a set of representatives for the $G_{\sigma^{-}}$ conjugacy classes of subgroups $a^{-1} N a$ of $G$ which are fixed by $\sigma$. Let $w_{i}=a_{i} a_{i}^{-\sigma} T$, so that $\left\{w_{i}\right\}$ is a set of representatives for the $\sigma$-conjugacy classes of $W$. Then

$$
\sum_{i} \frac{\varepsilon\left(w_{i}\right)}{\left|\left(a_{i}^{-1} N a_{i}\right)_{\sigma}\right|}=\frac{p^{m}}{\left|G_{\sigma}\right|}
$$

where $\varepsilon$ is the alternating character of $W$ and $p^{m}$ is the highest power of $p$ dividing $\left|G_{\sigma}\right|$.

Proof. $\sigma$ induces a linear transformation $\sigma^{*}$ of the real vector space $V$ generated by the character group of $T$. We may regard $W$ as acting on $V$. Let $I$ be the ideal of $W$-invariant elements without constant term in the algebra of polyno- 
mials on $V$, and $J=I / I^{2}$. Steinberg ([6], 11.19) has shown, if $G$ is semisimple, that

$$
\operatorname{det}\left(\sigma_{J}^{*}-1_{J}\right)^{-1}=\frac{p^{m}}{\left|G_{\sigma}\right|}
$$

We can extend this to the case when $G$ is reductive, as follows. Let $G^{\prime}$ be the derived group of $G$, so that $G^{\prime}$ is semisimple. Then $\left(G / G^{\prime}\right)_{\sigma} \cong\left(T / T \cap G^{\prime}\right)_{\sigma}$ and, as $G^{\prime}$ is connected, $G_{\sigma} / G_{\sigma}^{\prime} \cong\left(G / G^{\prime}\right)_{\sigma}([6], 10.11)$. Hence $\left(G_{\sigma}: G_{\sigma}^{\prime}\right)=\left|\left(T / T \cap G^{\prime}\right)_{\sigma}\right|$. Now $V=U \oplus V_{1}$ where $U\left(V_{1}\right)$ isomorphic to the real vector space generated by the character group of $T / T \cap G^{\prime}\left(T \cap G^{\prime}\right)$, and $W$ acts trivially on $U$. Hence

$$
\operatorname{det}\left(\sigma_{J}^{*}-1_{J}\right)^{-1}=\frac{p^{m}}{\left|G_{\sigma}^{\prime}\right|} \operatorname{det}\left(\sigma_{U}^{*}-1_{U}\right)^{-1} .
$$

However, by duality det $\left(\sigma_{U}^{*}-1_{U}\right)=\left|\left(T / T \cap G^{\prime}\right)\right|_{\sigma}$ and this yields the result.

Again, Steinberg $([6], 14.6)$ has shown that

$$
\frac{1}{|W|} \sum_{w \in W} \operatorname{det}\left(\sigma^{*} w-1\right)^{-1}=\operatorname{det}\left(\sigma_{J}^{*}-1_{J}\right)^{-1} .
$$

Suppose a maximal torus $a^{-1} \mathrm{Ta}$ of $G$ is fixed by $\sigma$ and let $n_{0}=a a^{-\sigma}$. Let $T_{0}=\left[t \in T \mid n_{0}^{-1} t n_{0}=t^{\sigma}\right]$. Clearly $\left(a^{-1} T a\right)_{\sigma}=a^{-1} T_{0} a$. Thus if $n_{0} T=w_{0}$ then $\left|T_{0}\right|=\left|\operatorname{Ker}\left(\sigma-w_{0}\right)\right|$ where $w_{0}$ is regarded as acting on $T$. But $\left|\operatorname{Ker}\left(\sigma-w_{0}\right)\right|=$ $\operatorname{det}\left(\sigma^{*}-w_{0}\right)$ where $w_{0}$ is regarded on the right hand side as acting on $V$. Thus, by (2.2), (2.3) and Lemma 4 we have

$$
\frac{p^{m}}{\left|G_{\sigma}\right|}=\sum_{i} \frac{\varepsilon\left(w_{i}\right)}{\left|Z^{\prime}\left(w_{i}\right)\right|} \operatorname{det}\left(\sigma^{*}-w_{i}\right)=\sum_{i} \frac{\varepsilon\left(w_{i}\right)}{\left|\left(a_{i}^{-1} N a_{i}\right)_{\sigma}\right|}
$$

This proves the lemma.

Remark. In the case when $G$ is defined and split over a field of $q$ elements and $\sigma$ is the $q^{\text {th }}$ power map, (2.3) follows from a classical formula of Molien (see eg [4]). In that case $\left|\left(a^{-1} T a\right)_{\sigma}\right|=f(q)$ where $f(x)$ is the characteristic polynomial of $w=a a^{-\sigma} T$ acting on $V$. See also [5], p. 62 .

\section{Preparatory results}

In this section our aim is to consider certain characters of the subgroups $\left(a_{i}^{-1} N a_{i}\right)_{\sigma}$ defined in Lemma 5 and the characters of $G_{\sigma}$ induced from them. The results given below are preparatory to computing the values of these induced characters.

Until the end of Lemma 7, $G$ satisfies the same assumptions as in $\S 2$. Let $n \in N$, then $n \in\left(a^{-1} N a\right)_{\sigma}$ if and only if $a a^{-\sigma} \in Z^{\prime \prime}(n)$. Suppose $Z^{\prime \prime}(n) \neq \phi$ and let $n_{0}$ be a fixed element of $Z^{\prime \prime}(n)$, so that $Z^{\prime \prime}(n)=Z(n) n_{0}$. If now $n^{\prime} \in Z(n)$ and $n_{1} \in Z^{\prime \prime}(n)$ then $n^{\prime} n_{1} n^{\prime-\sigma} \in Z^{\prime \prime}(n)$ showing that $Z(n)$ acts on $Z^{\prime \prime}(n)$ by $\sigma$-conjugation. 
Consider the equivalence relation $R$ on $Z^{\prime \prime}(n)$ given by

$$
\left(n_{1} n_{2}\right) \in R \text { if and only if } n_{1}=n_{2} t\left(\left(n_{1}, n_{2}\right) \in Z^{\prime \prime}(n) \times Z^{\prime \prime}(n)\right),
$$

where $t \in n_{0}^{-1}(Z(n) \cap T)_{0} n_{0}$. It is easy to see that the action of $Z(n)$ on $Z^{\prime \prime}(n)$ by $\sigma$-conjugation induces an action of the finite group $U=Z(n) /(Z(n) \cap T)_{0}$ on the finite set $B$ which is the quotient of $Z^{\prime \prime}(n)$ by the relation $R$.

LeMMA 6. The number of orbits of $U$ acting on $B$ is equal to the number of $(Z(n), \sigma)$-conjugacy classes of $Z^{\prime \prime}(n)$ i.e. the number of orbits of $Z(n)$ acting on $Z^{\prime \prime}(n)$ by $\sigma$-conjugation.

Proof. Suppose $n_{1}, n_{2} \in Z^{\prime \prime}(n)$ are such that for some $n^{\prime} \in Z(n)$ we have $\left(n_{2}, n^{\prime} n_{1} n^{-\sigma}\right) \in R$. Then we show that $n_{1}$ and $n_{2}$ are $\sigma$-conjugate by an element of $Z(n)$. We have $n_{2}=n^{\prime} n_{1} n^{\prime-\sigma} t$ where $t \in n_{0}^{-1}(Z(n) \cap T)_{0} n_{0}$. If $n_{2}=c c^{-\sigma}(c \in G)$ then $c^{-\sigma} t^{-1} c^{\sigma}=c^{-1} n^{\prime} n_{1} n^{\prime-\sigma} c^{\sigma}$. Now $n_{2} n_{0}^{-1} \in Z(n)$ and hence $c^{-\sigma} t^{-1} c^{\sigma} \epsilon$ $c^{-1}(Z(n) \cap T)_{0} c$. By arguing as in Lemma 2 we see that $n_{2}=t_{1}^{-1} n^{\prime} n_{1} n^{\prime-\sigma} t_{1}$ for some $t_{1} \in(Z(n) \cap T)_{0}$ and this proves the lemma.

We now suppose that the subgroups $a_{i}^{-1} N a_{i}(i=1,2, \cdots)$ are chosen as in Lemma 5.

LEMMA 7. Let $n \in N$ and suppose that $Z^{\prime \prime}(n) \neq \emptyset$. Then there exists a one-to-one correspondence between $(Z(n), \sigma)$-conjugacy classes of $Z^{\prime \prime}(n)$ and conjugacy classes of the $\left(a_{i}^{-1} N a_{i}\right)$ containing elements of the form $a_{i}^{-1} n^{\prime-1} n n^{\prime} a_{i}\left(n^{\prime} \in N\right)$. Furthermore, if $a_{i} a_{i}^{-\sigma}=n_{i}$, the order of the centraliser of $a_{i}^{-1} n^{\prime-1} n n^{\prime} a_{i}$ in $\left(a_{i}^{-1} N a_{i}\right)_{\sigma}$ is $\left|Z(n) \cap Z^{\prime}\left(n^{\prime} n_{i} n^{\prime-\sigma}\right)\right|$.

PRoof. We note that $a_{i}^{-1} n a_{i} \in\left(a_{i}^{-1} N a_{i}\right)_{\sigma}$ if an only if $a_{i} a_{i}^{-\sigma} \in Z^{\prime \prime}(n)$. Thus, by replacing the subgroups $a_{i}^{-1} N a_{i}$ by conjugates by elements of $G_{\sigma}$ if necessary, we see that to each $(N, \sigma)$-conjugacy class of $Z^{\prime \prime}(n)$ there corresponds a unique subgroup $\left(a_{i}^{-1} N a_{i}\right)_{\sigma}$ which contains $a_{i}^{-1} n a_{i}$. Choose a fixed such $i$, let $a_{i}=a$ and let $a a^{-\sigma}=n_{0} \in Z^{\prime \prime}(n)$. We show that each $(Z(n), \sigma)$-conjugacy class of $Z^{\prime \prime}(n)$ contained in the $(N, \sigma)$-conjugacy class of $n_{0}$ gives rise to a conugacy class of $\left(a^{-1} N a\right)_{\sigma}$ containing an element of the form $a^{-1} n^{\prime-1} n n^{\prime} a\left(n^{\prime} \in N\right)$ and conversely. Let $n_{1} \in Z^{\prime \prime}(n)$ be such that $n_{1}=n^{\prime} n_{0} n^{\prime-\sigma}$ where $n^{\prime} \in N$, Since $n_{1} \in Z^{\prime \prime}(n)$ we have $n_{1}^{-1} n n_{1}=n^{\sigma}$, but this is equivalent to $a^{-1} n^{\prime-1} n n^{\prime} a \in\left(a^{-1} N a\right)_{\sigma}$. Suppose $n_{1}$, $n_{2} \in Z^{\prime \prime}(n), n_{i}=n_{i}^{\prime} n_{0} n_{i}^{\prime-\sigma}\left(n_{i}^{\prime} \in N, i=1,2\right)$ and that $n_{1}=n^{\prime} n_{2} n^{\prime-\sigma}$ where $n^{\prime} \in Z(n)$. Then $x=a^{-1} n_{1}^{\prime-1} n^{\prime} n_{2}^{\prime \prime} a \in\left(a^{-1} N a\right)_{\sigma}$ and $a^{-1} n_{2}^{\prime-1} n n_{2} a=x^{-1} a^{-1} n_{1}^{\prime-1} n n_{1}^{\prime} a x$. Conversely if $a^{-1} n_{2}^{\prime-1} n n_{2} a=a^{-1} y^{-1} n_{1}^{\prime-1} n n_{1}^{\prime} y a$ where $a^{-1} y a \in\left(a^{-1} N a\right)_{\sigma}$ then $n_{1}=n^{\prime} n_{2} n^{\prime-\sigma}$ where $n^{\prime}=n_{1}^{\prime} y n_{2}^{\prime-1} \in Z(n)$. This proves the first part of the lemma.

Now let $a^{-1} n^{-1} n n^{\prime} a \in\left(a^{-1} N a\right)_{\sigma}$ and suppose $n^{\prime} n_{0} n^{\prime-\sigma}=b b^{-\sigma}(b \in G)$. Then $b^{-1} n^{\prime} a=g \in G_{\sigma}$ and $g a^{-1} n^{\prime-1} n n^{\prime} a g^{-1} \in\left(b^{-1} N b\right)_{\sigma}$. Thus the centraliser of $a^{-1} n^{\prime-1} n n^{\prime} a$ in $\left(a^{-1} N a\right)_{\sigma}$ is conjugate in $G_{\sigma}$ to the centraliser of $b^{-1} n b$ in 
$\left(b^{-1} N b\right)_{\sigma}$, and it is sufficient to show that the order of the centraliser of $a^{-1} n a$ in $\left(a^{-1} N a\right)_{\sigma}$ is $\left|Z(n) \cap Z^{\prime}\left(n_{0}\right)\right|$. But this is clear and the lemma is proved.

For the rest of this section we will assume that $G$ is simply connected and semisimple. For each subgroup $a^{-1} N a$ of $G$ which is fixed by $\sigma$ our aim is to define a certain linear character of $\left(a^{-1} N a\right)_{\sigma}$ which is trivial on $\left(a^{-1} T a\right)_{\sigma}$. Since each such subgroup is conjugate by an element of $G_{\sigma}$ to one of the $a_{i}^{-1} N a_{i}$, it is sufficient to define such characters of the $\left(a_{i}^{-1} N a_{i}\right)_{\sigma}$. For this it is sufficient, by Lemma 4 , to define a linear character $\psi_{w}$ of $Z^{\prime}(w)$ for each $w$ in a set of representatives for the $\sigma$-conjugacy classes of $w$.

Assume for the moment that we have chosen a linear character $\psi_{w_{i}}$ of $Z^{\prime}\left(w_{i}\right)$ (where, as before, $w_{i}=a_{i} a_{i}^{-\sigma} T$ ) and denote the corresponding character of $\left(a_{i}^{-1} N a_{i}\right)_{\sigma}$ also by $\psi_{w_{i}}$. Let

$$
\phi=\sum_{i} \varepsilon\left(w_{i}\right) \psi_{w_{i}}^{*}
$$

where $\psi_{w_{i}}^{*}$ is the character of $G_{\sigma}$ induced from $\psi_{w_{i}}$.

In order that $\phi$ is the Steinberg character of $G_{\sigma}$ the characters $\psi_{w_{i}}$ should satisfy certain conditions which will be stated in Lemma 8. Finally the characters will be defined under certain assumptions on $G$ in $\S 4$ and $\phi$ will then be proved to be the Steinberg character of $G_{\sigma}$.

Lemma 8. Let $p$ be prime to $|W|$. Suppose we have, for each $w \in W$, a linear character $\psi_{w}$ of $Z^{\prime}(w)$ such that these characters have the following properties. If $w_{2}=w^{\prime} w_{1} w^{\prime}-\sigma$ for some $w^{\prime} \in W$ (and hence $Z^{\prime}\left(w_{2}\right)=w^{\prime} Z^{\prime}\left(w_{1}\right) w^{\prime-1}$ ) then $\psi_{w_{1}}(w)=\psi_{w_{2}}\left(w^{\prime-1} w w^{\prime}\right)$ for any $w$ in $Z^{\prime}\left(w_{1}\right)$. Furthermore

$$
\begin{gathered}
\text { If } n \in N-T, Z^{\prime \prime}(n)=Z(n) n_{0}, Z(n) T / T=Y \subseteq W \text { and } \\
K(n) T / T=Y^{\prime} \subseteq Y \text {, then for each coset } Y^{\prime} c \text { of } Y^{\prime} \text { in } Y, \\
\sum_{w \in Y^{\prime} c w_{0}} \psi_{w}(z) \varepsilon(w)=0,
\end{gathered}
$$

where $n T=z, n_{0} T=w_{0}$.

Using these characters let $\phi$ be defined as in (3.2). Then the contribution to $\phi$ from the elements of the $\left(a_{i}^{-1} N a_{i}\right)_{\sigma}$ which lie outside $\left(a_{i}^{-1} T a_{i}\right)_{\sigma}$ is zero.

PROOF. Let $n \in N-T$ and let $n_{0}, w_{0}, Y, Y^{\prime}$ be as in the statement of the lemma. We will consider all the conjugacy classes of each $\left(a_{i}^{-1} N a_{i}\right)_{\sigma}$ which contain elements of the form $a_{i}^{-1} n^{\prime-1} n n^{\prime} a_{i}$ for some $n^{\prime} \in N$. Since $p$ is prime to $|W|$ these elements are semisimple. Hence, since these elements are all conjugate in $G$ and $G$ is simply connected they are conjugate in $G_{\sigma}([6], 12.5)$ to, say, $x \in G_{\sigma}$. We show that the contribution to $\phi(x)$ from all such classes is zero. Since the set of all conjugacy classes of the $\left(a_{i}^{-1} N a_{i}\right)_{\sigma}$ which are contained in the conjugacy class of $x$ in $G$ is a union of sets of classes of this type, the lemma will then be proved. 
We have the formula

$$
\phi(x)=\left|Z_{G_{\sigma}}(x)\right| \sum_{i} \varepsilon\left(w_{i}\right) \sum_{j} \frac{1}{\left|Z_{\left(a_{i}-1 N a_{i}\right)_{\sigma}}\left(n_{i j}\right)\right|} \psi_{w_{i}}\left(n_{i j}\right)
$$

where the second sum is over a set of representatives of conjugacy classes of $\left(a_{i}^{-1} N a_{i}\right)_{\sigma}$ containing elements conjugate to $x$ in $G_{\sigma}$. Thus the contribution to $\phi(x)$ from a class of $\left(a_{i}^{-1} N a_{i}\right)_{\sigma}$ containing an element of the form $a_{i}^{-1} n^{\prime-1} n n^{\prime} a_{i}$ is

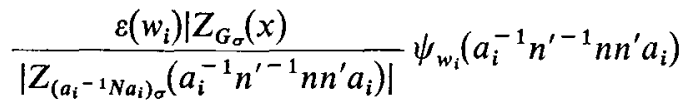

$$
\begin{aligned}
& =\frac{\varepsilon\left(w_{i}\right)\left|Z_{G_{\sigma}}(x)\right|}{\left|Z(n) \cap Z^{\prime}\left(n^{\prime} n_{i} n^{\prime-\sigma}\right)\right|} \psi_{w_{i}}\left(a_{i}^{-1} n^{\prime-1} n n^{\prime} a_{i}\right) \text {, }
\end{aligned}
$$

where $n_{i}=a_{i} a_{i}^{-\sigma}$, by Lemma 7 .

Furthermore, let $n^{\prime} T=w^{\prime}$. Then $\psi_{w_{i}}\left(a_{i}^{-1} n^{\prime-1} n n^{\prime} a_{i}\right)=\psi_{w_{i}}\left(w^{\prime-1} w w^{\prime}\right)=$ $\psi_{w^{\prime} w_{i} w^{\prime}-\sigma}(w)$, since $w^{\prime-1} Z^{\prime}\left(w^{\prime} w_{i} w^{\prime-\sigma}\right) w^{\prime}=Z^{\prime}\left(w_{i}\right)$. We note that in the one-to-one correspondence given by Lemma 7 , the class of $\left(a_{i}^{-1} N a_{i}\right)_{\sigma}$ containing $a_{i}^{-1} n^{\prime-1} n n^{\prime} a_{i}$ corresponds to the $(Z(n), \sigma)$-class of $Z^{\prime \prime}(n)$ containing $n^{\prime} n_{i} n^{\prime-\sigma}$. Thus the required contribution to $\phi(x)$ from the classes of the $\left(a_{i}^{-1} N a_{i}\right)_{\sigma}$ containing elements of the form $a_{i}^{-1} n^{\prime-1} n n^{\prime} a_{i}$ is

$$
\left|Z_{G_{\sigma}}(x)\right| \sum_{n^{\prime \prime}} \frac{\varepsilon\left(w^{\prime \prime}\right)}{\left|Z(n) \cap Z^{\prime}\left(n^{\prime \prime}\right)\right|} \psi_{w^{\prime \prime}}(z)
$$

where the summation is over a set of representatives of the $(Z(n), \sigma)$-conjugacy classes of $Z^{\prime \prime}(n)$, and $n^{\prime \prime} T=w^{\prime \prime}$.

Now let $U, B$ be as in Lemma 6 , and for $n^{\prime \prime} \in Z^{\prime \prime}(n)$ let $b^{\prime \prime} \in B$ be the residue class of $n^{\prime \prime}$ and $C\left(b^{\prime \prime}\right) \subseteq U$ the stabilizer of $b^{\prime \prime}$ under the action of $U$ on $B$. We show that the natural map of $Z(n) \cap Z^{\prime}\left(n^{\prime \prime}\right)$ into $C\left(b^{\prime \prime}\right)$ is surjective. Indeed, let $n_{1} \in Z(n)$ be such that $n^{\prime \prime}=n_{1} n^{\prime \prime} n_{1}^{-\sigma} t$ for some $t \in(Z(n) \cap T)_{0}$. An argument similar to that of Lemma 2 yields that $n^{\prime \prime}=t_{1}^{-1} n_{1} n^{\prime \prime} n_{1}^{-\sigma} t_{1}^{\sigma}$ and hence that $t_{1}^{-1} n_{1} \in Z(n) \cap Z^{\prime}\left(n^{\prime \prime}\right)$ for some $t_{1} \in(Z(n) \cap T)_{0}$. Thus (3.5) can be written as

$$
\left|Z_{G_{\sigma}}(x)\right| \sum_{n^{\prime \prime}} \frac{\varepsilon\left(w^{\prime \prime}\right)}{\left|(Z(n) \cap T)_{0} \cap Z^{\prime}\left(n^{\prime \prime}\right)\right|\left|C\left(b^{\prime \prime}\right)\right|} \psi_{w^{\prime \prime}}(z) .
$$

Since, by Lemma 6, the number of $(Z(n), \sigma)$-classes of $Z^{\prime \prime}(n)$ is also the number of orbits of $U$ acting on $B$, this expression is equal to

$$
\frac{\left|Z_{G_{\sigma}}(x)\right|}{|U|} \sum_{n^{\prime \prime}} \frac{\varepsilon\left(w^{\prime \prime}\right)}{\left|(Z(n) \cap T)_{0} \cap Z^{\prime}\left(n^{\prime \prime}\right)\right|} \psi_{w^{\prime \prime}}(z)
$$

where now the summation is over a set of representatives from the equivalence classes of $Z^{\prime \prime}(n)$ given by (3.1). Since $\left(Z(n) \cap T:(Z(n) \cap T)_{0}\right)$ is finite we can rewrite this expression as 


$$
\frac{\left|Z_{G_{\sigma}}(x)\right|}{|Y|} \sum_{w^{\prime \prime}} \frac{\varepsilon\left(w^{\prime \prime}\right)}{\left|(Z(n) \cap T)_{0} \cap Z^{\prime}\left(n^{\prime \prime}\right)\right|} \psi_{w^{\prime \prime}}(z)
$$

where the sum is over a set of representatives for $Y w_{0}$ in $Z^{\prime \prime}(n)$.

For any two elements $n_{1}, n_{2} \in Z^{\prime \prime}(n),(Z(n) \cap T)_{0} \cap Z^{\prime}\left(n_{1}\right)=(Z(n) \cap T)_{0}$ $\cap Z^{\prime}\left(n_{2}\right)$ if and only if $K(n) n_{1}=K(n) n_{2}$. Hence the above expression will be zero if for each coset $Y^{\prime} c$ of $Y^{\prime}$ in $Y$

$$
\sum_{w \in Y^{\prime} c w_{0}} \varepsilon(w) \psi_{w}(z)=0 .
$$

But this is the assumption of the lemma, and the lemma is proved.

The next lemma is concerned with elements of the $\left(a_{i}^{-1} N a_{i}\right)_{\sigma}$ which lie in $\left(a_{i}^{-1} T a_{i}\right)_{\sigma}$.

Lemma 9. Let $a^{-1}$ Ta be a maximal torus of $G$ fixed by $\sigma$, let $s \in\left(a^{-1} T a\right)_{\sigma}$ and $H=Z_{G}(s)$. Then there exists a one-to-one correspondence between the conjugacy classes of $\left(a^{-1} \mathrm{Na}\right)_{\sigma}$ which contain elements of $\left(a^{-1} \mathrm{Ta}\right)_{\sigma}$ that are conjugate to $\mathrm{s}$ in $G_{\sigma}$ and the $H_{\sigma}$-conjugacy classes of maximal tori of $H$ which are fixed by $\sigma$ and are $G_{\sigma}$-conjugate to $a^{-1} T a$.

Proof. Since $G$ is simply connected, $H$ is a connected reductive group by a theorem of Steinberg $([6], 8.2)$. The maximal tori of $H$ are precisely those maximal tori of $G$ which contain $s$. It follows then that the element $g^{-1} s g\left(g \in G_{\sigma}\right)$ lies in $\left(a^{-1} T a\right)_{\sigma}$ if and only if $g a^{-1} \mathrm{Tag}^{-1} \subseteq H$. Furthermore, two elements $g_{1}^{-1} s g_{1}$ and $g_{2}^{-1} s g_{2}\left(g_{1}, g_{2} \in G_{\sigma}\right)$ are conjugate in $\left(a^{-1} N a\right)_{\sigma}$ if and only if $g_{1} a^{-1} T_{a g} g_{1}^{-1}$ and $g_{2} a^{-1} \mathrm{Tag}_{2}^{-1}$ are conjugate by an element of $H_{\sigma}$, as was to be shown.

\section{The main theorem}

We now state the main theorem. The notation is that of $\S 2$.

THEOREM. Let $G$ be simply connected and semisimple. Suppose $p$ does not divide $|W|$ and let the subgroups $a_{i}^{-1} N a_{i}(i=1,2, \cdots)$ be chosen as in Lemma 5 . For each $w \in W$ suppose we have a linear character $\psi_{w}$ of $Z^{\prime}(w)$ such that these characters have the properties described in Lemma 8, and let $\phi$ be then defined by (3.2). Then $\phi$ is the Steinberg character of $G_{\sigma}$. Thus, if $s$ is a semisimple element of $G_{\sigma}$ and $H=Z_{G}(s)$ then $\phi(s)=\varepsilon(s) n(s)$ where $n(s)$ is the order of a Sylow p-subgroup of $H_{\sigma}$ and $\varepsilon(s)= \pm 1$ has the following interpretation. Let $Q$ be a maximal torus of $H$ such that $Q$ and a Borel subgroup of $H$ containing $Q$ are both fixed by $\sigma$. Regard $Q$ as a maximal torus of $G$, and suppose the element $w(s)$ of $W$ corresponds to $Q$ (in the sense of the remarks following Lemma 3). Then $\varepsilon(s)=\varepsilon(w(s))$.

Proof. Let $s$ be a semisimple element of $G_{\sigma}$. By Lemma 8 it is sufficient to consider the contributions to $\phi(s)$ from elements of the form $g^{-1} s g \in\left(a_{i}^{-1} T a_{i}\right)_{\sigma}$ $\left(g \in G_{\sigma}\right)$. The order of the centraliser of $g^{-1} s g$ in $\left(a_{i}^{-1} N a_{i}\right)_{\sigma}$ is 


$$
\left|\left(a_{i}^{-1} N a_{i}\right)_{\sigma} \cap Z_{G}\left(g^{-1} s g\right)\right|=\left|\left(g a_{i}^{-1} N a_{i} g\right)_{\sigma} \cap H\right|=\left|\left(g a_{i}^{-1} N a_{i} g \cap H\right)_{\sigma}\right|
$$

Hence

$$
\phi(s)=\left|H_{\sigma}\right| \sum_{i} \varepsilon\left(w_{i}\right) \sum_{g} \frac{1}{\left|\left(g a_{i}^{-1} N a_{i} g \cap H\right)_{\sigma}\right|}
$$

where the second sum is over a set of representatives of the conjugacy classes of $\left(a_{i}^{-1} N a_{i}\right)_{\sigma}$ containing elements of the form $g^{-1} s g\left(g \in G_{\sigma}\right)$. Under the correspondence mentioned in Lemma 9, the conjugacy class of $g^{-1} s g$ in $\left(a_{i}^{-1} N a_{i}\right)_{\sigma}$ corresponds to the $H_{\sigma}$-conjugacy class of the maximal torus $g^{-1} a_{i}^{-1} T a_{l} g$ of $H$. Using this correspondence we see that

$$
\phi(s)=\left|H_{\sigma}\right| \sum_{c^{-1} T c} \frac{\varepsilon\left(c c^{-\sigma} T\right)}{\left|\left(c^{-1} N c \cap H\right)_{\sigma}\right|}
$$

where the sum is over a set of representatives of the $H_{\sigma}$-conjugacy classes of maximal tori of $H$ fixed by $\sigma$.

Consider the maximal torus $Q$ of $H$ and let $Q=x^{-1} T x(x \in G)$. Let $\varepsilon^{\prime}$ be the alternating character of $x^{-1} N x / x^{-1} T x$; thus if $y \in N$ then $\varepsilon^{\prime}\left(x^{-1} y x Q\right)=$ $\varepsilon(y T)$. By Lemma 5 applied to the connected reductive group $H$ we have

$$
\sum_{b^{-1} Q b} \frac{\varepsilon^{\prime}\left(b b^{-\sigma} Q\right)}{\left|\left(b^{-1} x^{-1} N x b \cap H\right)_{\sigma}\right|}=\frac{n(s)}{\left|H_{\sigma}\right|}
$$

where again the sum is over a set of representatives of the $H_{\sigma}$-conjugacy classes of maximal tori of $H$ fixed by $\sigma$. Now

$$
\begin{aligned}
\varepsilon\left(x b b^{-\sigma} x^{-\sigma} T\right) & =\varepsilon^{\prime}\left(b b^{-\sigma} x^{-\sigma} x Q\right)=\varepsilon^{\prime}\left(b b^{-\sigma} Q\right) \varepsilon^{\prime}\left(x^{-\sigma} x Q\right) \\
& =\varepsilon^{\prime}\left(b b^{-\sigma} Q\right) \varepsilon\left(x x^{-\sigma} T\right)=\varepsilon^{\prime}\left(b b^{-\sigma} Q\right) \varepsilon(w(s)) .
\end{aligned}
$$

By (4.1) and (4.2) we than get $\phi(s)=\varepsilon(w(s)) n(s)$ as required.

Finally, $\phi$ is zero at elements of $G_{\sigma}$ which are not semisimple. For any semisimple element $s$ the absolute value of $\phi(s)$ is the absolute value of the value of Steinberg character of $G_{\sigma}$ at $s$. This shows that $\phi$ is irreducible. Since the degree of $\phi$ is the order of a Sylow $p$-subgroup of $G_{\sigma}, \phi$ is the Steinberg character of $G_{\sigma}$. This proves the theorem.

\section{REMARKS}

1. We note that the condition that $p$ does not divide $|W|$ was used in Lemma 8 to derive the following result.

$$
\text { Let } n \in N-T \text { be such that } Z^{\prime \prime}(n) \neq \phi \text {. }
$$

If $a_{i}^{-1} n^{\prime-1} n n^{\prime} a_{i} \in\left(a_{i}^{-1} N a_{i}\right)_{\sigma}$ and $a_{j}^{-1} n^{\prime \prime-1} n n^{\prime \prime} a_{j} \in\left(a_{j}^{-1} N a_{j}\right)_{\sigma}$ for some $i, j$ and $n^{\prime}$, $n^{\prime \prime} \in N$, then $a_{i}^{-1} n^{\prime-1} n n^{\prime} a_{i}$ and $a_{j}^{-1} n^{\prime \prime-1} n n^{\prime \prime} a_{j}$ are conjugate in $G_{\sigma}$. Thus the condition that $p$ does not divide $|W|$ can be dispensed with if $(4.3)$ is satisfied in $G_{\sigma}$.

2. Suppose $G$ is defined and split over $k=G F(q)$ and $\sigma$ is the $q^{\text {th }}$ power map. 
Then $T$ is a $k$-split maximal torus of $G$. In this case, for a semisimple element $s$, $w(s)$ is the element of $W$ corresponding to a maximal torus of $H$ containing a maximal $k$-split torus of $H$, when regarded as a maximal torus of $G$. If $s$ is regular then $H$ is a maximal torus of $G$ and $w(s)$ is the element of $W$ corresponding to $H$.

3. The characters we have defined of the subgroups $\left(a^{-1} \mathrm{Na}\right)_{\sigma}$ are all trivial on $\left(a^{-1} T a\right)_{\sigma}$. It is likely that this can be modified as follows. Take any character $\eta$ of $T$ and let $N^{\prime}$ be the subgroup of $N$ which fixes $\eta$. If characters of the subgroups $\left(a^{-1} N^{\prime} a\right)_{\sigma}$ can be defined which have properties similar to (3.3) and which take the value $\eta(t)$ at an element $a^{-1} t a$ of $\left(a^{-1} T a\right)_{\sigma}$ then these could be used to construct characters of $G_{\sigma}$ analogous to $\phi$. For example, suppose $\eta$ is a character of $T$ which is not fixed by any $w \neq 1$ of $W$. For each $w \in W$ choose $a \in G$ such that $a a^{-\sigma} T=w$ and let $\eta_{w}$ be the character defined by $\eta_{w}\left(a^{-1} t a\right)=\eta(t)$ of $\left(a^{-1} T a\right)_{\sigma}$. Let $\chi=\Sigma_{w \in W} \varepsilon(w) \eta_{w}^{*}$. Then $\chi$ is a character of $G_{\sigma}$ of degree $|W| n(1)$. In this case we need not put any restriction on $p$ as Lemma 8 is not used. It seems likely that the characters of $G$ obtained in this way are some of the principal indecomposable characters (for $p$ ) of $G_{\sigma}$.

\section{Construction of certain characters of subgroups of $\boldsymbol{W}$}

In this section we give illustrations of when the main theorem can be applied, by actually constructing the characters $\psi_{w}$ in certain cases.

Assume that $G$ is simple. We have the following possibilities for $G_{\sigma}[6]$ :

(1) $G$ is of type $A_{l}, B_{l}, C_{l} \cdots, E_{8}$ and $G_{\sigma}$ is a Chevalley group.

(2) (i) $G$ is of type $A_{l}(l \geqq 2), D_{l}(l \geqq 4)$ or $E_{6}$ and $G_{\sigma}$ is a twisted analogue of a Chevalley group.

(ii) $G$ is of type $D_{4}$ and $G_{\sigma}$ is a 'triality form' of $G$.

(3) (i) $G$ is of type $B_{2}, p=2$ and $G_{a}$ is Suzuki group.

(ii) $G$ is of type $G_{2}$ and $p=3$ or $G$ is of type $F_{4}$ and $p=2$ and $G_{\sigma}$ is a Ree group.

In the case when $W=W_{\sigma}$ we have $Z^{\prime}(w)=Z(w)=Z^{\prime \prime}(w)$ for each $w \in W$. Then we have to define characters $\psi_{w}$ of $Z(w)$ for each $w \in W$ having the property (3.3) where we may take $w_{0}=1$. In Case $1, W=W_{\sigma}$. In Case 2 we make the following remark. Suppose there is an element $w^{\prime}$ in $W$ such that $w^{\prime-1} w w^{\prime}=w^{\sigma}$ for all $w \in W$. This means that $Z^{\prime}\left(w^{\prime}\right)=W$. By Lemma 4 there is a maximal torus $a^{-1} \mathrm{Ta}$ of $G$ fixed by $\sigma$ such that $\left(a^{-1} \mathrm{Na}\right)_{\sigma} /\left(a^{-1} \mathrm{Ta}\right)_{\sigma}$ is isomorphic to $W$. Let $T^{\prime}=a^{-1} T a, N^{\prime}=a^{-1} N a, N^{\prime} / T^{\prime}=W^{\prime}$, so that $W^{\prime}=W_{\sigma}^{\prime}$. Suppose we replace $T$ by $T^{\prime}$, and $N$ by $N^{\prime}$ and define the required characters $\psi_{w}$ on subgroups $Z^{\prime}(w)$ of $W^{\prime}\left(w \in W^{\prime}\right)$. Then the expression on the left side of (3.4) will be replaced by the same expression where the elements are now taken form $W^{\prime}$, possibly multiplied by -1 since $T^{\prime}$ corresponds to the element $a a^{-\sigma} T$ of $W$. Hence in this 
case we can assume that $W=W_{\sigma}$ and define the characters $\psi_{w}$ as in Case 1 . We remark that $W$ contains such an element $w^{\prime}$ if $G$ is of type $A_{l}, D_{l}\left(l\right.$ odd) or $E_{6}$.

In Case 3 the characteristic of $K$ divides $|W|$ and so Lemma 8 cannot be used. However, in the case of types $B_{2}$ and $G_{2}$, if we suitably define the characters $\psi_{w}$ and then define $\phi$ as in (3.2), we can check directly using the results of Suzuki [8] and Ward [9] that $\phi$ is the Steinberg character of $G_{\sigma}$.

(5.1) The subgroup $Y$ in Lemma 8 depends on the choice of $n$ and not merely on $z=n T \in W$. However, suppose $z$ lies in a direct product $W^{\prime}$ of reflection subgroups of $W$, each subgroup being generated by reflections corresponding to a closed set of roots (with respect to $T$ ) of $G$. For a root $\alpha$ let $Z_{\alpha}$ be the centraliser in $G$ of the connected component of the kernel of $\alpha$. As $\alpha$ runs over the roots considered above, let $H$ be the reductive subgroup of $G$ generated by the $Z_{\alpha}$. Then $W^{\prime}$ is the Weyl group of $H$ and $n \in H$. From the structure of $H([1], 17)$ it follows that if $z=z_{1} z_{2} \cdots$ according to the decomposition of $W^{\prime}$, there exist elements $n_{1}, n_{2}, \cdots$ in $Z(n)$ such that $n_{1} T=z_{1}, n_{2} T=z_{2}, \cdots$. Hence $Y$ contains at least the cyclic groups generated by $z_{1}, z_{2}, \cdots$. This fact will be used later.

In order to define the characters $\psi_{w}$ we consider in turn the possible types for $W$. The groups $W$ for each of the possible types for $G$ are described in ([1], 19). In Cases (i), (ii) and (iii) $\sigma$ is the identity automorphism of $W$.

(i) Type $A_{l} . W$ is the symmetric group $S_{l+1}$. Let $z \in W$ be written as a product of disjoint cycles. Then $K(z)$ is the direct product of the cyclic groups generated by these cycles. By applying the argument of (4.1) we see that $K(z) \subseteq Y$. Hence it is sufficient to show (3.4) with $Y^{\prime}=K(z)$. The structure of $Z(z)$ is well known and can be described as follows. Let $z=w_{1} w_{2} \cdots$ where $w_{m}=\left(b_{1}^{\prime} b_{2}^{\prime} \cdots b_{m}^{\prime}\right)$ $\left(b_{1}^{2} b_{2}^{2} \cdots b_{m}^{2}\right) \cdots\left(b_{1}^{r} b_{2}^{r} \cdots b_{m}^{r}\right)$ is the product of those cycles occuring in $z$ which are of length $m$. Let $T_{m}$ be the group $V_{1} V_{2} \cdots V_{r} M$ where $V_{i}$ is the cyclic subgroup generated by $b_{i}=\left(b_{1}^{i} b_{2}^{i} \cdots b_{m}^{i}\right)$, and $M$ is generated by elements of $W$ of the form $c_{1} c_{2} \cdots c_{m}$ where $c_{1}$ is a permutation of $b_{1}^{\prime}, \cdots b_{1}^{r}$ and $c_{i}$ is got from $c_{1}$ by replacing $b_{1}^{j}$ by $b_{i}^{j}$. The $Z(z)=T_{1} \times T_{2} \times \cdots \times T_{m} \times \cdots$. Define the character $\psi_{z}$ of $Z(z)$ by putting $\psi_{z}(y)=1$ for all $y$ in $M$, and defining it on $T_{m}$ by $\psi_{z}\left(b_{i}\right)=\theta$, where, if $m=2^{a} k$ ( $k$ odd) then $\theta$ is a primitieve $k^{\text {th }}$ root of unity.

It can then be verified that these characters satisfy (3.4).

(ii) Types $B_{l}(l \geqq 2), C_{l}(l \geqq 3)$. $W$ is a semidirect product of a normal subgroup $P$ which is elementary abelian of order $2^{l}$ generated by reflections $a_{1}$, $a_{2}, \cdots a_{l}$, with a subgroup $W_{0}$ which is generated by reflections $(1 i)(i=2, \cdots, l)$. $W_{0}$ is isomorphic to $S_{l}$ and we have

$$
(1 i) a_{j}(1 i)=\left\{\begin{array}{l}
a_{j} \text { if } j \neq i, 1 \\
a_{1} \text { if } j=i \\
a_{i} \text { if } j=1
\end{array}\right.
$$


The conjugacy classes of $W$ are described, e.g. in [10].

As in (i) we can show that if $z \in W$ then $K(z) \subseteq Y$. Suppose $z \in W_{0}$ is of the form

$$
\left(b_{1}^{\prime} b_{2}^{\prime} \cdots b_{m}^{\prime}\right) \cdots\left(b_{1}^{r} b_{2}^{r} \cdots b_{m}^{r}\right)
$$

as in (i). Then we define the character $\psi_{z}$ of $Z(z)$ by defining $\psi_{z}$ on $Z(z) \cap W_{0}$ as in (i) and by stipulating that $\psi_{z}(y)=1$ for $y$ in $Z(z) \cap P$. Now suppose

$$
z=a_{\alpha_{1}}\left(b_{1}^{\prime} b_{2}^{\prime} \cdots b_{m}^{\prime}\right) a_{\alpha_{2}}\left(b_{1}^{2} b_{1} \cdots b_{m}^{2}\right) \cdots a_{\alpha_{r}}\left(b_{1}^{r} b_{2}^{r} \cdots b_{m}^{r}\right),
$$

where $\alpha_{i}=b_{1}^{i}$. Let $V_{i}$ be the cyclic group generated by $b_{i}=a_{\alpha_{i}}\left(b_{1}^{i} b_{2}^{i} \cdots b_{m}^{i}\right)$ and $M$ the subgroup of $Z(z)$ defined as in (i). Define $\psi_{z}$ on $A_{1} A_{2} \cdots A_{r} M$ by $\psi_{z}(y)=1$ for $y$ in $M$ and $\psi_{z}\left(b_{i}\right)=\theta$ where $\theta$ is a primitive $m^{\text {th }}$ root of unity.

We can then define the characters $\psi_{z}$ for a general element $z$, and it can be verified that they have the required property.

(iii) Type $D_{l}(l \geqq 4)$. The Weyl group $W^{\prime}$ in this case is the subgroup of index 2 of the group $W$ of (ii) consisting of all elements of the form $a_{1}^{\delta_{1}} a_{2}^{\delta_{2}} \cdots a_{l}^{\delta_{1} y}$ $\left(y \in W_{0}\right)$ where $\Sigma \delta_{i}$ is even. If two elements of $W^{\prime}$ are such that the corresponding elements $y$ in $W_{0}$ fix at least one symbol from 1 to $l$ they are conjugate in $W^{\prime}$ if and only if they are conjugate in $W$. However, if $x=a_{1}^{\delta_{1}} \cdots a_{l}^{\delta_{l}} y$ and $y$ moves all the symbols from 1 to $l$ then the conjugacy class of $x$ in $W$ splits into two classes in $W^{\prime}$. For any element $z \in W$ we now define a character $\psi_{z}$ of its centraliser in $W$. If $z \in W^{\prime}$, the required character of its centraliser in $W^{\prime}$ is defined to be the restriction of this character. If $z$ is of the form (5.2) we define $\psi_{z}$ as in (ii). If $z$ is of the form (5.3) define $\psi_{z}(y)=1$ for $y \in M$ and $\psi_{z}\left(b_{i}\right)=\theta$ where $\theta$ is a primitive $(2 \mathrm{~m})^{\text {th }}$ root of unity.

We remark that in this case it is not clear whether we always have, for $z \in W, K(z) \subseteq Y$. However, using the remarks (5.1) we can again verify that the characters $\psi_{z}$ have the required properties.

(iv) Type $D_{l}(l \geqq 4)$, with $\sigma$ the automorphism of $W^{\prime}$ which interchanges (12) and $a_{1} a_{2}(12)$. By the remarks at the beginning of the section we can assume that $l$ is even. We regard $W^{\prime}$ as a subgroup of the Weyl group $W$ of the type $D_{l+1}$ in the obvious way. Since $l+1$ is odd there is an element $w_{0} \in W$ (in fact $w_{0}=$ $\left.a_{2} a_{3} \cdots a_{l+1}\right)$ such that $w_{0}^{-1} w w_{0}=w^{\sigma}$ for all $w \in W$. Then for any $w \in W$, $Z^{\prime}(w)=Z\left(w w_{0}^{-1}\right)$. Thus if $z \in W^{\prime}, Z^{\prime}(z) \cap W^{\prime} \subseteq Z\left(z w_{0}^{-1}\right)$. In order to define the character $\psi_{z}$ of $Z^{\prime}(z) \cap W^{\prime}$ we define it on $Z\left(z w_{0}^{-1}\right)$ as in (iii) and restrict it to $Z^{\prime}(z) \cap W^{\prime}$. These characters again have the required properties.

(v) Type $D_{4}$, with $\sigma$ the automorphism of $W^{\prime}$ which maps (12) $\rightarrow a_{1} a_{2}(12)$, $a_{1} a_{2}(12) \rightarrow(34),(34) \rightarrow(12)$. The $\sigma$-conjugacy classes of $W^{\prime}$ can be calculated. We give in the colums of table 1 from left to right, representatives $z$ of the classes, $Z^{\prime}(z)$, and the characters to be chosen. We put $x=a_{1} a_{2} a_{3} a_{4}$. 
TABLE 1

\begin{tabular}{lll}
\hline 1 & $\left\{a_{1} a_{2}(234),(23)\right\}$ & Trivial Character \\
$x$ & $\left\{a_{1} a_{2}(234),(23)\right\}$ & Trivial Character \\
$(12)$ & $\left\{x, a_{3} a_{4}(34)\right\}$ & Trivial Character \\
$a_{2} a_{3}(12)$ & $\{x,(12)\}$ & Trivial Character \\
$a_{3} a_{4}(123)$ & $\left\{(123), a_{1} a_{4}(12)(34)\right\}$ & $(123) \rightarrow \omega, a_{1} a_{4}(12)(34) \rightarrow 1$ \\
$(123)$ & $\left\{a_{1} a_{4}(12)(34)\right\}$ & $a_{1} a_{4}(12)(34) \rightarrow-1$ \\
$a_{1} a_{2}(123)$ & $\left\{(123), a_{1} a_{4}(12)(34)\right\}$ & $(123) \rightarrow \omega, a_{1} a_{4}(12)(34) \rightarrow 1$ \\
\hline
\end{tabular}

Here, and in the rest of the section, $\omega$ and $i$ denote a primitive cube root and a fourth root of unity respectively.

(vi) Type $G_{2} . W$ is generated by reflections $b$ and $c$ such that $a=b c$ is of order 6 .

1. $\sigma$ is the identity. Let $\psi_{a}$ be the character $a \rightarrow \omega$ of $Z(a)=\{a\}$. For the centralisers of elements of $W$ not conjugate to $a$ we take the trivial character.

2. $\sigma$ is the automorphism which interchanges $b$ and $c$. We have $Z^{\prime}(1)=\left\{a^{3}\right\}$, $Z^{\prime}(b)=Z^{\prime}\left(a^{2} b\right)=Z^{\prime}\left(a^{3} b\right)=\{a\}$. We choose the trivial character of $Z^{\prime}(1)$ $Z^{\prime}(b)$ and the character $a \rightarrow \omega$ of $Z^{\prime}\left(a^{2} b\right)$ and $Z^{\prime}\left(a^{3} b\right)$.

(vii) Type $B_{2}, W$ is generated by two reflections $b$ and $c$ such that $a=b c$ is of order 4. Let $\sigma$ be the automorphism interchanging $b$ and $c$. We have $Z^{\prime}(1)=$ $\left\{a^{2}\right\}, Z^{\prime}(b)=Z^{\prime}\left(a^{2} b\right)=\{a\}$. Choose the character $a^{2} \rightarrow-1$ of $Z^{\prime}(1)$ and the character $a \rightarrow i$ of $Z^{\prime}(b)$ and $Z^{\prime}\left(a^{2} b\right)$.

(viii) Type $F_{4}, W$ is a group of order 1152 , the conjugacy classes and characters of which are given in [2]. $\sigma$ is the identity automorphism of $W$. It can be shown, by a lengthy computation, that there exist characters $\psi_{w}$ of $Z(w)$ for $w \in W$ such that $\psi_{w^{\prime}}(w)=\psi_{w}\left(w^{\prime}\right)$ for any pair $w, w^{\prime} \in W$. These characters have the required properties.

In the case of the Weyl group of type $E_{6}$ we can define characters $\psi_{w}$ having properties (3.3) provided $Y^{\prime}=K(z)$ in (3.4). However, we have not been able to show that $Y^{\prime}=K(z)$ holds for each $z$.

Summarizing, the main theorem is valid in the following cases.

(1) $G$ is of type $A_{l}, B_{l}, C_{l}, D_{l}, G_{2}, F_{4}$ and $G_{\sigma}$ is a Chevalley group.

(2) $G$ is of type $A_{l}, D_{l}$ and $G_{\sigma}$ is a twisted form of a Chevalley group.

(3) $G$ is of type $D_{4}$ and $G_{a}$ is a triality form of $G$.

(4) $G_{\sigma}$ is a Suzuki group or a Ree group of type $G_{2}$.

\section{References}

[1] C. Chevalley, Seminaire sur la classification des groups deLie algebriques (Paris, 1958).

[2] K. Kondo, 'The characters of the Weyl group of type $F_{4}$ ', Jour. Fac. Sci. Univ. of Tokyo $11(1965), 145-153$. 
[3] S. Lang, 'Algebraic groups over finite fields', Amer. J. Math. 78 (1956), 555-563.

[4] G. C. Shephard, and J. A. Todd, 'Finite unitary reflection groups', Canadian J. Math. 6 (1954), 274-304.

[5] L. Solomon, 'Invariants of finite reflection groups', Nagoya Math. J. 22 (1963), 57-64.

[6] R. Steinberg, Endomorphism of linear algebraic groups (Memoirs of the American Math. Socitty, No. 80).

[7] R. Steinberg, 'Representations of algebraic groups', Nagoya Math. J. 22 (1963), 33-56.

[8] M. Suzuki, 'On a class of doubly transitive groups', Annals of Math. 75 (1962), 105-145.

[9] H. N. Ward, 'On Ree's series of simple groups', Trans. Amer. Math. Soc. 121 (1966), 62-89.

[10] M. Osima, 'On the representations of the generalized symmetric group', Math. J. Okayama Univ. 4 (1954), 39-55.

Added in proof. A description of the one-to-one correspondence mentioned after Lemma 1 is contained in

[11] T. A. Springer and R. Steinberg, Seminar on Algebraic Groups and Related Finite Groups (Lecture Notes in Mathematics, No. 131, Springer-Verlag, 1970).

The Ramanujan Institute

University of Madras

Madras-5, India

and

Clark University 